\title{
Primary cutaneous aggressive epidermotropic cytotoxic T-cell lymphomas: reappraisal of a provisional entity in the 2016 WHO classification of cutaneous lymphomas
}

Joan Guitart ${ }^{1}$, M Estela Martinez-Escala ${ }^{1}$, Antonio Subtil ${ }^{2}$, Madeleine Duvic ${ }^{3}$, Melissa P Pulitzer ${ }^{4}$, Elise A Olsen ${ }^{5}$, Ellen Kim ${ }^{6}$, Alain H Rook ${ }^{6}$, Sara S Samimi ${ }^{6}$, Gary S Wood ${ }^{7}$, Michael Girardi ${ }^{2}$, Jacqueline Junkins-Hopkins ${ }^{8}$, Doina S Ivan ${ }^{3}$, M Angelica Selim ${ }^{5}$, Kimberly A Sable ${ }^{1}$, Pooja Virmani ${ }^{5}$, Laura B Pincus ${ }^{9}$, Michael T Tetzlaff ${ }^{3}$, Jinah $\mathrm{Kim}^{10}$ and Youn H Kim ${ }^{10}$

${ }^{1}$ Department of Dermatology and Pathology, Northwestern University Feinberg Medical School, Chicago, IL, USA; ${ }^{2}$ Department of Dermatology and Pathology, Yale University, New Haven, CT, USA; ${ }^{3}$ Departments of Dermatology and Pathology, MD Anderson, Houston, TX, USA; ${ }^{4}$ Department of Dermatology and Pathology, Sloan Kettering Cancer Center, New York, NY, USA; ${ }^{5}$ Department of Dermatology and Pathology, Duke University, Durham, NC, USA; ${ }^{6}$ Department of Dermatology and Pathology, University of Pennsylvania, Philadelphia, PA, USA; ${ }^{7}$ Department of Dermatology and Pathology, University of Wisconsin VAMC, Madison, WI, USA; ${ }^{8}$ Department of Dermatology and Pathology, Ackerman Academy, New York, NY, USA; ${ }^{9}$ Department of Dermatology and Pathology, University of California in San Francisco, San Francisco, CA, USA and ${ }^{10}$ Department of Dermatology and Pathology, Stanford University, Stanford, CA, USA

Primary cutaneous CD8-positive aggressive epidermotropic T-cell lymphoma is a rare and poorly characterized variant of cutaneous lymphoma still considered a provisional entity in the latest 2016 World Health Organization Classification of Cutaneous lymphomas. We sought to better characterize and provide diagnostic and therapeutic guidance of this rare cutaneous lymphoma. Thirty-four patients with a median age of 77 years (range 19-89 years) presented primarily with extensive annular necrotic plaques or tumor lesions with frequent mucous membrane involvement. The 5 -year survival was $32 \%$ with a median survival of 12 months. A subset of 17 patients had a prodrome of chronic patches prior to the development of aggressive ulcerative lesions. We identified cases with lack of CD8 or $\alpha \beta$ T-cell receptor expression yet with similar clinical and pathological presentation. Allogeneic stem cell transplantation provided partial or complete remissions in 5/6 patients. We recommend the term primary cutaneous aggressive epidermotropic cytotoxic T-cell lymphoma as this more broad designation better describes this clinical-pathologic presentation, which allows the inclusion of cases with CD8 negative and/or $a \beta / \gamma \delta$ T-cell receptor chain double-positive or double-negative expression. We have identified early skin signs of chronic patch/plaque lesions that are often misdiagnosed as eczema, psoriasis, or mycosis fungoides. Our experience confirms the poor prognosis of this entity and highlights the inefficacy of our standard therapies with the exception of allogeneic stem cell transplantation in selected cases.

Modern Pathology (2017) 30, 761-772; doi:10.1038/modpathol.2016.240; published online 27 January 2017

Primary cutaneous CD8-positive aggressive epidermotropic T-cell lymphoma remains a provisional entity in the latest World Health Organization

Correspondence: Dr J Guitart, MD, Department of Dermatology and Pathology, Northwestern University Feinberg School of Medicine 676N, St Clair street, Suite 1600, Chicago, IL 60611, USA.

E-mail: j-guitart@northwestern.edu

Received 19 September 2016; revised 15 December 2016; accepted 19 December 2016; published online 27 January 2017
2016 classification of cutaneous lymphomas. ${ }^{1}$ In part, the problem with CD8-positive aggressive epidermotropic T-cell lymphoma is that they have been poorly characterized to date owing to the rarity of the condition. Historically, this condition was known in the dermatology literature as generalized pagetoid reticulosis or Ketron-Goodman disease and was characterized by rapidly evolving and extensively ulcerated annular plaques associated with a poor prognosis. The histopathology has been 
reported to be characteristic, with an infiltrate of monomorphous medium-sized atypical lymphocytes involving the epidermal thickness in a so-called pagetoid pattern. Although clinically this condition was known to result in widespread ulcerations from epidermal necrosis, the cytotoxic phenotype with CD8 expression was first reported by Agnarsson et al. in 1990, and the term CD8-positive aggressive epidermotropic T-cell lymphoma was first coined by Berti et al. in 1999..$^{2,3}$ We have observed cases with similar clinical-pathologic features, cytotoxicity, and aggressive course without CD8 expression. Our goal is to redefine this entity using the more broad and inclusive term primary cutaneous aggressive epidermotropic cytotoxic T-cell lymphoma (PCAECyTCL). Herein we present the largest series of primary cutaneous aggressive epidermotropic cytotoxic T-cell lymphoma reported to date, collected from several cutaneous lymphoma centers in the United States.

\section{Materials and methods}

A group of pathologists and dermatologists from some of the largest cutaneous lymphoma centers in the United States met on two occasions in Chicago to review each institutional experience including the clinical and pathological material of primary cutaneous aggressive epidermotropic cytotoxic T-cell lymphoma. Approval from the Institutional Review Board at Northwestern University encompassing all centers was obtained. Entering criteria included a clinical presentation with patches, plaques, or nodules with erosive or necrotic features covering $>1 \%$ of the body surface area. Cases with localized pagetoid reticulosis (Woringer-Kolopp disease) were excluded. The cases included were characterized by (1) a pagetoid pattern of T-cells with expression of at least one pan-T-cell marker (2) positivity for CD8 and $\beta F 1$ ( $\alpha \beta$ T-cell receptor heterodimer) and (3) negativity for the $\gamma \delta$ T-cell receptor heterodimer marker (clone 73.20 , Thermo/Fisher Scientific, Waltham, MA, USA). However, these criteria were revised when we encountered cases that fulfilled clinicopathologic criteria but with variable CD8 and/or T-cell receptor immunophenotype. An extensive immunohistochemistry panel of monoclonal antibodies against CD2, CD3, CD4, CD5, CD7, CD8, CD20, CD30, CD45RA, CD56, T-cell intracellular antigen-1, and granzyme B was performed in most cases. In addition Epstein-Barr virus ribonucleic acid expression was evaluated using Epstein-Barr encoding region in situ hybridization and $\mathrm{T}$-cell receptor clonality analysis with polymerase chain reaction based methods using BIOMED-2 protocol was reported when available from skin, blood, nodal, and bone marrow biopsy samples.

Electronic medical records were reviewed and clinical, imaging, and laboratory parameters along with clinical photographs when available were presented to the group. Clinical data including gender, age, race, nature, extent and duration of the lesions, constitutional symptoms, past medical history, results of staging bone marrow biopsy and imaging tests, response to therapies, outcome, and duration of follow-up were obtained. The original histopathological slides including immunohistochemical markers were reviewed by the group and a consensus diagnosis was rendered. Skin biopsies from the 30 selected patients were reviewed. Several parameters including the distribution of the infiltrate within the epidermal, dermal and subcutaneous compartments, distribution of the intraepidermal lymphocytes, cell size, adnexotropic features (folliculotropism and syringotropism), presence or absence of epidermal necrosis, interface reaction, ulceration, hemorrhage, angiocentricity, or vasculitis were assessed by the group on a multiheaded microscope. Other notable histological findings such as presence of other inflammatory cells (eosinophils, neutrophils, plasma cells, histiocytes), granuloma formation, mucin deposits, karyorrhexis, or hemophagocytosis were documented.

Descriptive statistics including median (with range) and counts (with percentages) were determined and compared using $U$ Mann - Whitney and Fisher's Exact tests, respectively. Five-year survival rate was performed using Kaplan-Meier method. Categorical clinical and pathological characteristics were compared using log-rank tests to overall outcome and survival rates. Survival analysis was performed from date of diagnosis. As this was a retrospective analysis, data were missing for some cases. All analyses were done on the available data. All tests were performed using SPSS statistics software (Chicago, IL, USA) with a type I error rate of $5 \%$. $P$-values $<0.05$ were considered indicative of a trend.

\section{Results}

\section{Clinical Data}

Upon a consensus review of a total of 52 cases, 30 were deemed to fulfill the diagnostic criteria and confirmed by the group as primary cutaneous aggressive epidermotropic cytotoxic T-cell lymphoma and 4 additional cases were added to the series over the ensuing months (Table 1). One case had been previously published as a case report. ${ }^{4}$ All cases were collected between 1995 and 2015. Most of the cases excluded were redefined after reviewing clinical and histological parameters by the group as CD8-positive mycosis fungoides, pagetoid reticulosis or dermal CD8-positive T-cell lymphomas without marked epidermotropism or pagetoid features.

The cohort consisted of 34 patients (25 male, 9 female) with a median age of 77 years (range 19-89 years) including 19 White, 5 Blacks, 4 Hispanic, 1 Asian, and 5 of unknown race. The median duration 
Table 1 Clinical summary of the cohort

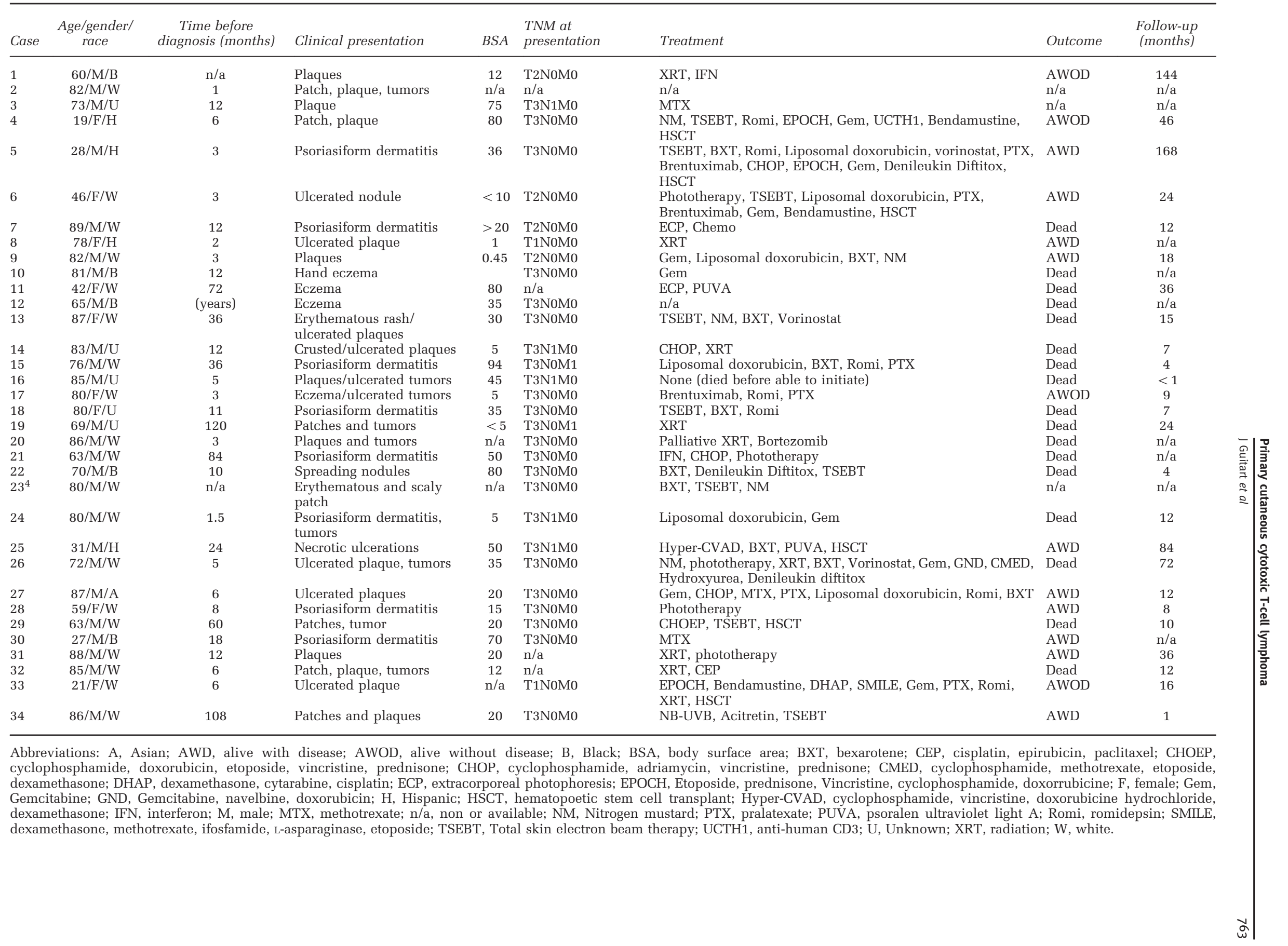



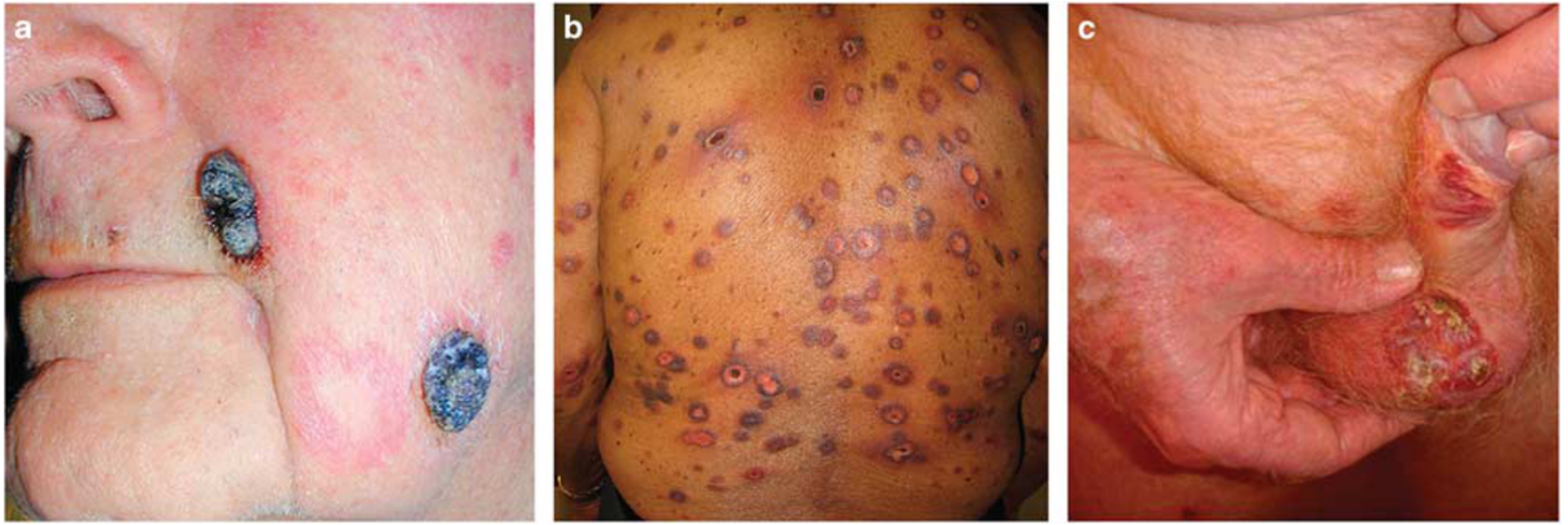

Figure 1 Clinical presentation of PCAECyTCL in three different patients. (a) Tumor presentation with exophytic hemorrhagic and necrotic nodules resembling pyogenic granuloma (Case 24). (b) Extensive targetoid necrotic macules and plaques resembling severe pityriasis lichenoides acuta or erythema multiforme (Case 27). (c) Ulcerated plaques involving genitalia (Case 23).

of the lesions at the time of presentation was 10 months, ranging from 1 to 120 . In 14 of 31 subjects the onset of symptoms was considered acute appearing within 6 months from the time of presentation. Seventeen patients had a chronic course longer than 6 months with poorly characterized preceding skin lesions. There was no survival difference from the time of diagnosis between those with an acute vs chronic course $(P=0.09)$. The skin lesions at presentation were reported as patches or plaques in 24 patients, tumors in 2 patients (Figure 1a, Case 24) and a combination of both in 7 patients. Ulceration was noted at presentation in 10 cases and 20 additional cases evolved into ulcerated plaques overtime. Thirteen patients carried an initial diagnosis of 'mycosis fungoides', whereas many other cases were initially diagnosed with a benign condition including an eczematous process, contact dermatitis, viral or drug eruption, psoriasis (11 cases), lupus erythematosus (2 cases), erythema multiforme or, ulcer. Two patients presented with acute coalescing necrotic macules resembling extensive pityriasis lichenoides acuta (Mucha-Habermann disease) (Figure 1b, Case 27). We also reviewed skin biopsies from two patients with the preceding non-specific skin presentations. In both cases rare scattered intraepidermal atypical lymphocytes were noted accompanied with acanthosis, hyperkeratosis and rare necrotic keratinocytes. Using the TNM classification system for primary cutaneous lymphomas other than mycosis fungoides and Sézary syndrome, multifocal or generalized skin involvement at presentation (T3) was reported in 24 patients. ${ }^{5}$ Two patients presented with a solitary lesion (T1) and four patients with localized involvement in one anatomic region (T2). Disease progression requiring systemic therapy was reported in all patients except in one with limited disease. The skin lesions were for the most part annular and erythematous with peripheral erosions and ulceration often surmounted with a hemorrhagic-necrotic crust (Figures 2a-c, Case 25). The ulcerated patches tended to become more generalized and extensive in advanced stages (Figures $2 \mathrm{~d}$ and e, Case 26). The distribution of the lesions was variable with involvement of the upper and/or lower extremities in 29 patients, torso in 23, and head/neck in 15 patients. Five patients developed ulcers involving the oral cavity and six patients involving genital or perigenital skin (Figure 1c, Case 23 and 2a, Case 25). Constitutional symptoms were overall mild with a few patients reporting weight loss (6 patients), lowgrade fever (3 patients), and asthenia (3 patients). Past medical history was significant for three patients with solid organ cancer (uterine, prostate, melanoma). One of these patients also had Parkinson disease, and one patient had a remote history of Hodgkin lymphoma in remission. Only one patient was immunosuppressed post-lung transplant and one patient had an unconfirmed diagnosis of bullous pemphigoid. Serum lactate dehydrogenase levels were increased at the time of presentation $(>200 \mathrm{U} / \mathrm{l})$ in $26 \%(5 / 19)$ of the patients. Four patients had abnormal peripheral blood results by flow cytometry including one patient with CD8+/CD26+ leukemia of 75000 cells/ml. The same patient was the only one $(1 / 11)$ with positive bone marrow biopsy. Small palpable lymphadenopathy was noted in six cases, but none of them were considered clinically significant for nodal biopsy. Clinical or pathological signs of hemophagocytosis or hepatosplenomegaly were not reported in any of the cases.

Results from imaging studies at presentation were available for 25 patients and were reported positive in 11 patients, most commonly demonstrating positron emission tomography avid cutaneous or subcutaneous uptake with only 1 patient with nodal involvement and 1 patient with right adrenal involvement. 

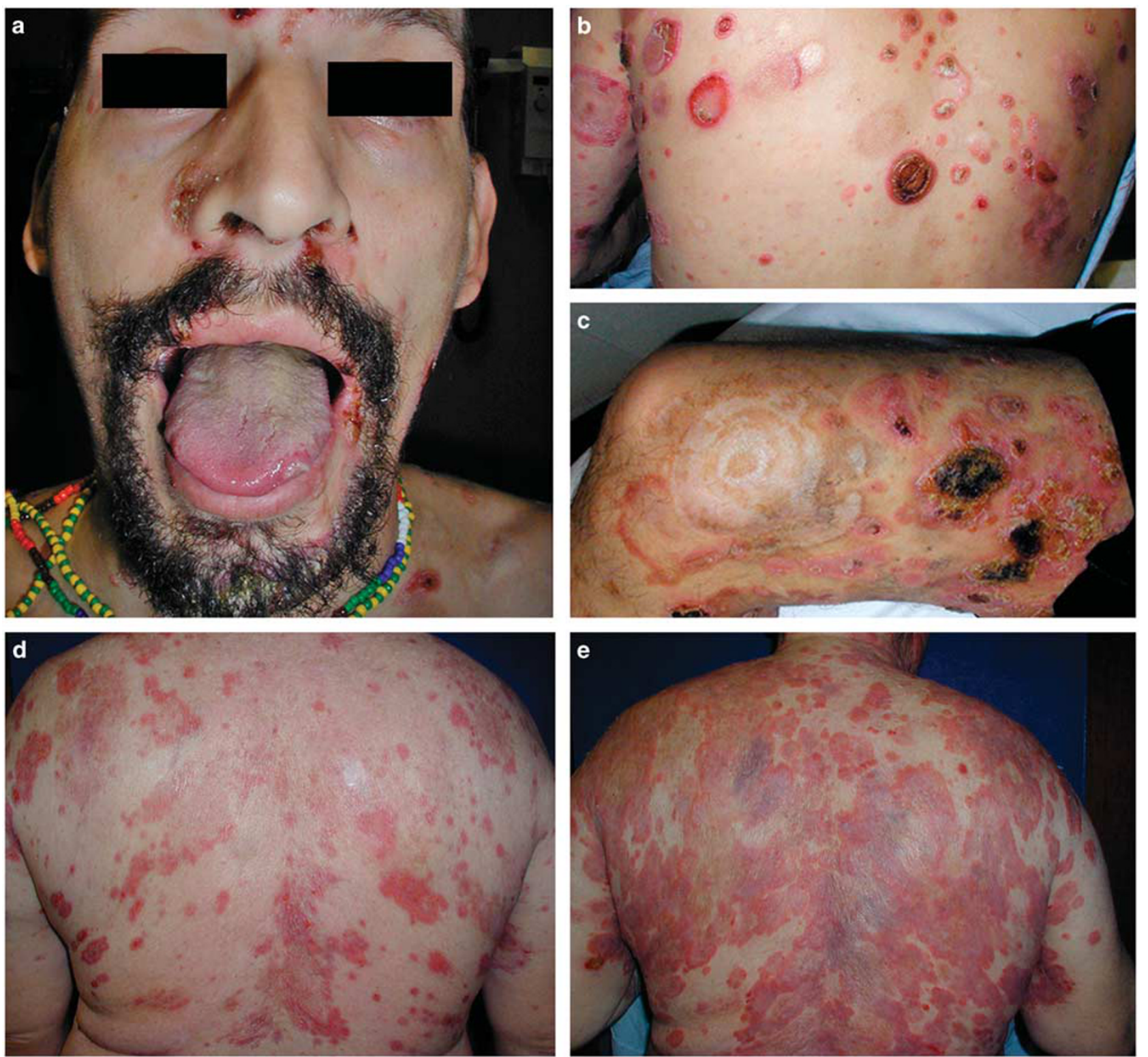

Figure 2 Clinical presentation of PCAECyTCL in two different patients. (a) Oral and nasal ulcerated macules (Case 25). (b) Extensive annular erosive plaques some with hemorrhagic features (Case 25). (c) Annular and arcuate ulcerated plaques and scars at sites of resolved lesions (Case 25). (d) Annular erythematous plaques at presentation (Case 26). (e) Same patient as 2D now with extensive erosive confluent plaques a few months later (Case 26).

Follow-up information was available for 31 patients with a median follow-up time of 12 months (range 1-168 months). Seventeen patients (54\%) died during the follow-up period related to disease progression. Four patients developed extracutaneous disease involving lungs (2 patients) and adrenal glands ( 2 patients). None of the patients developed hemophagocytic syndrome. At the last follow-up, 12 patients were known to be alive with disease, three alive without disease. The 5-year overall survival rate was $32 \%$ with a median overall survival of 12 months.

A median of three therapies (range 1-13), including skin-directed and systemic regimens, were adminis- tered to the cohort. Eight of the 20 patients treated with various chemotherapies were alive at the end of the study. Ten patients received localized radiation therapy and eight patients total skin electron beam therapy. Six patients were treated with allogeneic hematopoietic stem cell transplantation and five of them were alive at the end of the study including two in complete remission and three with recurrent disease albeit mostly limited to a few sporadic lesions. One patient developed extensive extracutaneous disease shortly after allogeneic hematopoietic stem cell transplantation while on immunosuppressive medication (detailed summary of therapies is presented in Table 2). 
Table 2 Clinical outcomes of radiation and systemic therapies

\begin{tabular}{|c|c|c|c|}
\hline Therapeutic agents & $\begin{array}{l}\text { Number of patients who } \\
\text { received the agent }(\%)\end{array}$ & $\begin{array}{l}\text { Number of patients who achieved any } \\
\text { response including } C R, P R\end{array}$ & $\begin{array}{c}\text { Significance regarding survival } \\
\text { outcome, } \mathrm{P} \text {-value }\end{array}$ \\
\hline Etoposide & $6(21)$ & 2 & 0.59 \\
\hline Gemcitabine & $9(30)$ & 3 & 0.13 \\
\hline Local radiation & $10(33)$ & 5 & 0.60 \\
\hline TSEBT & $8(26)$ & 6 & 0.56 \\
\hline Bexarotene (oral) & $9(30)$ & 1 & 0.56 \\
\hline Romidepsin & $6(21)$ & 1 & 0.16 \\
\hline Liposomal doxorubicin & $6(21)$ & 2 & 0.24 \\
\hline $\begin{array}{l}\text { Allogeneic stem cell } \\
\text { transplant }\end{array}$ & $6(21)$ & 5 & 0.06 \\
\hline
\end{tabular}

Abbreviations: CR, complete remission; PR, partial response; TSEB, total skin electron beam.

None of the treatments were significantly associated with a good outcome.

Table 3 Cytomorphology and immunophenotype of skin biopsies

\begin{tabular}{|c|c|c|c|c|c|c|c|c|c|c|c|c|c|c|c|}
\hline Cases & $\begin{array}{l}\text { Cell } \\
\text { size }\end{array}$ & $C D 2$ & CD3 & $C D 4$ & CD5 & $C D 7$ & CD8 & CD30 & CD45RA & CD56 & BetaF1 & TIA-1 & Granz B & $E B V$ & GM1 \\
\hline 1 & $\mathrm{~m} / \mathrm{l}$ & + & + & $-1+$ & $\stackrel{+/-}{\operatorname{dim}}$ & + & + & - & + & - & + & + & + & - & $\mathrm{n} / \mathrm{a}$ \\
\hline 2 & $\mathrm{~s} / \mathrm{m} / \mathrm{l}$ & - & + & - & - & - & + & - & + & - & + & + & $-1+$ & - & $\mathrm{n} / \mathrm{a}$ \\
\hline 3 & $\mathrm{~s} / \mathrm{m}$ & $\mathrm{n} / \mathrm{a}$ & + & - & - & + & + & - & + & + & - & + & n/a & - & - \\
\hline 4 & $\mathrm{~m} / \mathrm{l}$ & - & + & - & + & + & + & - & + & - & + & + & + & - & - \\
\hline 5 & $\mathrm{~m} / \mathrm{l}$ & - & Partial+ & - & - & + & + & $\begin{array}{l}+ \text { in } \\
\text { LC } \\
\text { cells }\end{array}$ & $\mathrm{n} / \mathrm{a}$ & - & + & + & + & - & - \\
\hline 6 & $\mathrm{~s} / \mathrm{m}$ & - & + & - & + & - & + & - & $\mathrm{n} / \mathrm{a}$ & - & + & + & $\mathrm{n} / \mathrm{a}$ & - & $\mathrm{n} / \mathrm{a}$ \\
\hline 7 & $\mathrm{~s} / \mathrm{m}$ & - & + & - & + & + & - & - & + & - & + & + & + & - & - \\
\hline 8 & $\mathrm{~s} / \mathrm{m}$ & - & + & - & - & - & + & $\begin{array}{c}\text { Weak } \\
30 \%\end{array}$ & + weak & - & - & + & $\mathrm{n} / \mathrm{a}$ & - & - \\
\hline 9 & $\mathrm{~m} / \mathrm{l}$ & - & + & - & - & + & + & - & $\mathrm{n} / \mathrm{a}$ & - & $\mathrm{n} / \mathrm{a}$ & + & + & - & $\mathrm{n} / \mathrm{a}$ \\
\hline 10 & $\mathrm{~s} / \mathrm{m} / \mathrm{l}$ & $\mathrm{n} / \mathrm{a}$ & + & $\mathrm{n} / \mathrm{a}$ & Rare & $\mathrm{n} / \mathrm{a}$ & + & $\mathrm{n} / \mathrm{a}$ & - & $30 \%$ & + & $\mathrm{n} / \mathrm{a}$ & $\mathrm{n} / \mathrm{a}$ & - & - \\
\hline 11 & $\mathrm{~m}$ & $\mathrm{n} / \mathrm{a}$ & + & - & $\mathrm{n} / \mathrm{a}$ & $\mathrm{n} / \mathrm{a}$ & + & - & $\mathrm{n} / \mathrm{a}$ & - & + & $\mathrm{n} / \mathrm{a}$ & + & $\mathrm{n} / \mathrm{a}$ & $\mathrm{n} / \mathrm{a}$ \\
\hline 12 & $\mathrm{~m} / \mathrm{l}$ & $\mathrm{n} / \mathrm{a}$ & + & - & - & $\mathrm{n} / \mathrm{a}$ & + & $\mathrm{n} / \mathrm{a}$ & $\mathrm{n} / \mathrm{a}$ & $\mathrm{n} / \mathrm{a}$ & + & + & + & - & n/a \\
\hline 13 & $\mathrm{~s} / \mathrm{m}$ & - & + & - & - & + & + & - & - & - & + & + & - & - & - \\
\hline 14 & $\mathrm{~m} / \mathrm{l}$ & - & + & - & - & + & + & - & Subset & - & + & + & - & - & - \\
\hline 15 & $\mathrm{~m} / \mathrm{l}$ & $-1+$ & + & - & + & + & + & - & + & - & + & - & - & - & - \\
\hline 16 & $\mathrm{~m}$ & + & + & - & + & + & + & - & - & - & + & + & Subset & $\mathrm{n} / \mathrm{a}$ & - \\
\hline 17 & $\mathrm{~m}$ & - & + & - & - & - & - & - & - & - & + & Subset & + & $\mathrm{n} / \mathrm{a}$ & - \\
\hline 18 & $\mathrm{~s} / \mathrm{m}$ & + & + & - & - & - & - & - & + & - & - & + & + & - & - \\
\hline 19 & $\mathrm{~s} / \mathrm{m}$ & $\mathrm{n} / \mathrm{a}$ & + & - & - & + & + & - & - & - & - & + & + & - & - \\
\hline 20 & $\mathrm{~m}$ & - & + & - & - & - & + & - & + & - & + & + & + & - & - \\
\hline 21 & $\mathrm{~s} / \mathrm{m}$ & + & + & - & - & - & + & - & - & - & - & + & - & - & - \\
\hline 22 & $\mathrm{~m} / \mathrm{l}$ & $\mathrm{n} / \mathrm{a}$ & + & - & - & + & + & - & $\mathrm{n} / \mathrm{a}$ & - & + & + & + & - & + \\
\hline $23^{4}$ & $\mathrm{~m} / \mathrm{l}$ & $\mathrm{n} / \mathrm{a}$ & + & - & - & + & + & - & + & - & + & + & - & - & - \\
\hline 24 & l & $\mathrm{n} / \mathrm{a}$ & + & - & - & + & + & $\begin{array}{c}50 \% \\
\exp \end{array}$ & Subset & + & + & + & + & - & $\mathrm{n} / \mathrm{a}$ \\
\hline 25 & $\mathrm{~s} / \mathrm{m}$ & $\mathrm{n} / \mathrm{a}$ & + & - & - & + & + & - & + & - & + & + & - & - & - \\
\hline 26 & $\mathrm{~s}$ & $\mathrm{n} / \mathrm{a}$ & + & - & + & + & + & - & $\mathrm{n} / \mathrm{a}$ & - & + & + & + & - & n/a \\
\hline 27 & $\mathrm{~s} / \mathrm{m}$ & - & + & - & + & + & + & - & + & - & + & + & + & - & + \\
\hline 28 & $\mathrm{~s}$ & + & + & + (intraep) & + & - & + (intraep) & - & - & - & + & $\begin{array}{c}+ \\
\text { (intraep), } \\
\text { subset }\end{array}$ & $\begin{array}{c}+ \\
\text { (intraep), } \\
\text { subset }\end{array}$ & - & - \\
\hline 29 & $\mathrm{~m}$ & $\mathrm{n} / \mathrm{a}$ & + & - & - & + & - & $\mathrm{n} / \mathrm{a}$ & + & - & + & + (golgi) & + & $\mathrm{n} / \mathrm{a}$ & - \\
\hline 30 & $\mathrm{~s} / \mathrm{m}$ & + & + & - & + & + & - & - & - & - & - & + & + & - & - \\
\hline 31 & $\mathrm{~m} / \mathrm{l}$ & - & + & - & - & + & Weak & - & $\mathrm{n} / \mathrm{a}$ & - & + & + & + & - & $\mathrm{n} / \mathrm{a}$ \\
\hline 32 & $\mathrm{~s} / \mathrm{m} / \mathrm{l}$ & + & + & - & + & + & - & $30 \%$ & - & - & + & + & - & - & - \\
\hline 33 & $\mathrm{~s} / \mathrm{m}$ & + & + & - & - & + & Weak & - & + & + & Weak & + & + & - & - \\
\hline & $\mathrm{s} / \mathrm{m}$ & + & + & - & - & + & - & $\mathrm{n} / \mathrm{a}$ & - & - & + & \pm & $\mathrm{n} / \mathrm{a}$ & $\mathrm{n} / \mathrm{a}$ & - \\
\hline$\%(+)$ & $\begin{array}{l}41 \% \\
\text { of LC }\end{array}$ & 43 & 100 & 6 & 33 & 74 & 79 & 13 & 61 & 12 & 82 & 97 & 76 & 100 & 12 \\
\hline
\end{tabular}

Abbreviations: EBV, Epstein-Barr virus; Granz B, granzyme B; intraep, intraepidermal; l, large; LC, large; m, medium; n/a, not available; s, small; TIA-1, T-cell intracellular antigen-1. 


\section{Pathology Data}

Thirty-four biopsies were assessed. The pattern of the lymphoid infiltrate was pagetoid in all cases with scattered atypical lymphocytes involving the entire epidermal thickness in 20 biopsies, whereas in 14 biopsies the atypical lymphocytes were predominantly confined to the lower half of the epidermis
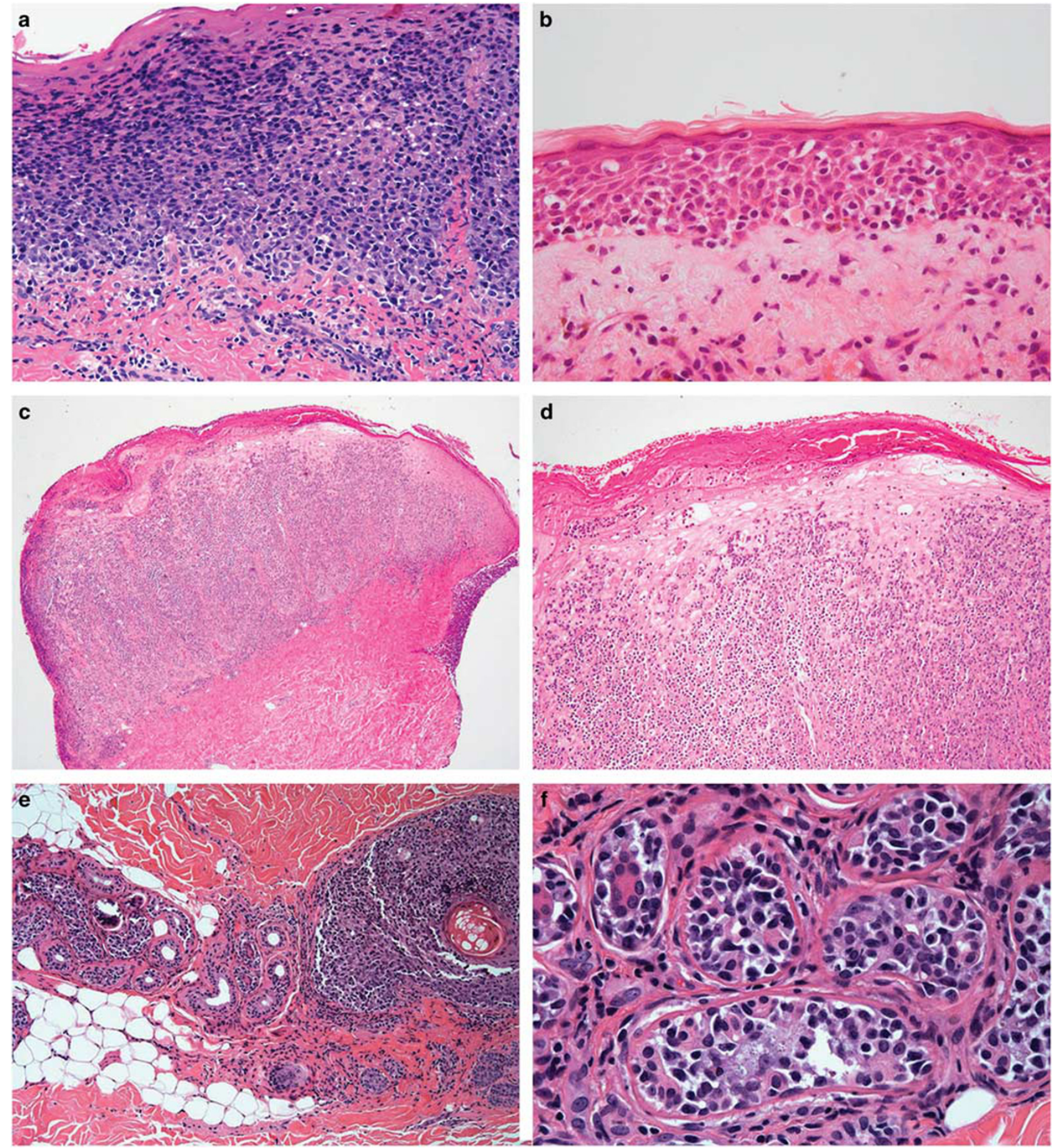

Figure 3 Histopathological features of PCAECyTCL. (a) H\&E stains showing extensive full-thickness pagetoid exocytosis of medium-sized lymphocytes without significant keratinocytes necrosis or dermal involvement (Case 25). (b) Atrophic epidermis with pagetoid intraepidermal atypical small/medium lymphocytes mostly involving the lower levels of the epidermis. Occasional necrotic keratinocytes are noted (Case 34). (c) Tumor lesion with a predominantly dermal infiltrate (Case 29). (d) Same as C in higher magnification showing extensive pagetoid exocytosis in addition to a dense dermal infiltrate (Case 29). (e) Marked lymphoid infiltration of eccrine coils on the left side and pilosebaceous unit on the right side (Case 26). (f) High power of image e demonstrating atypical small lymphocytes in the eccrine coil (Case 26). 
(Figures 3a-d Case 25, Case 34, and Case 29). The latter pattern was commonly associated with an atrophic epidermis. Infiltration of cutaneous adnexa by the lymphoma cells was common, but eccrine glands or follicular units were absent from some skin biopsies (Figures 3e and f, Case 26). Syringotropism of the eccrine glands without hyperplastic changes was noted in 18/30 biopsies and folliculotropism in $18 / 22$. However, follicular mucinosis or destruction of the follicular unit by the tumor cells as commonly seen in folliculotropic mycosis fungoides were not observed in any case. In addition to the intraepithelial infiltrate, nine biopsies had a tumoral dermal component and in six additional biopsies the infiltrate
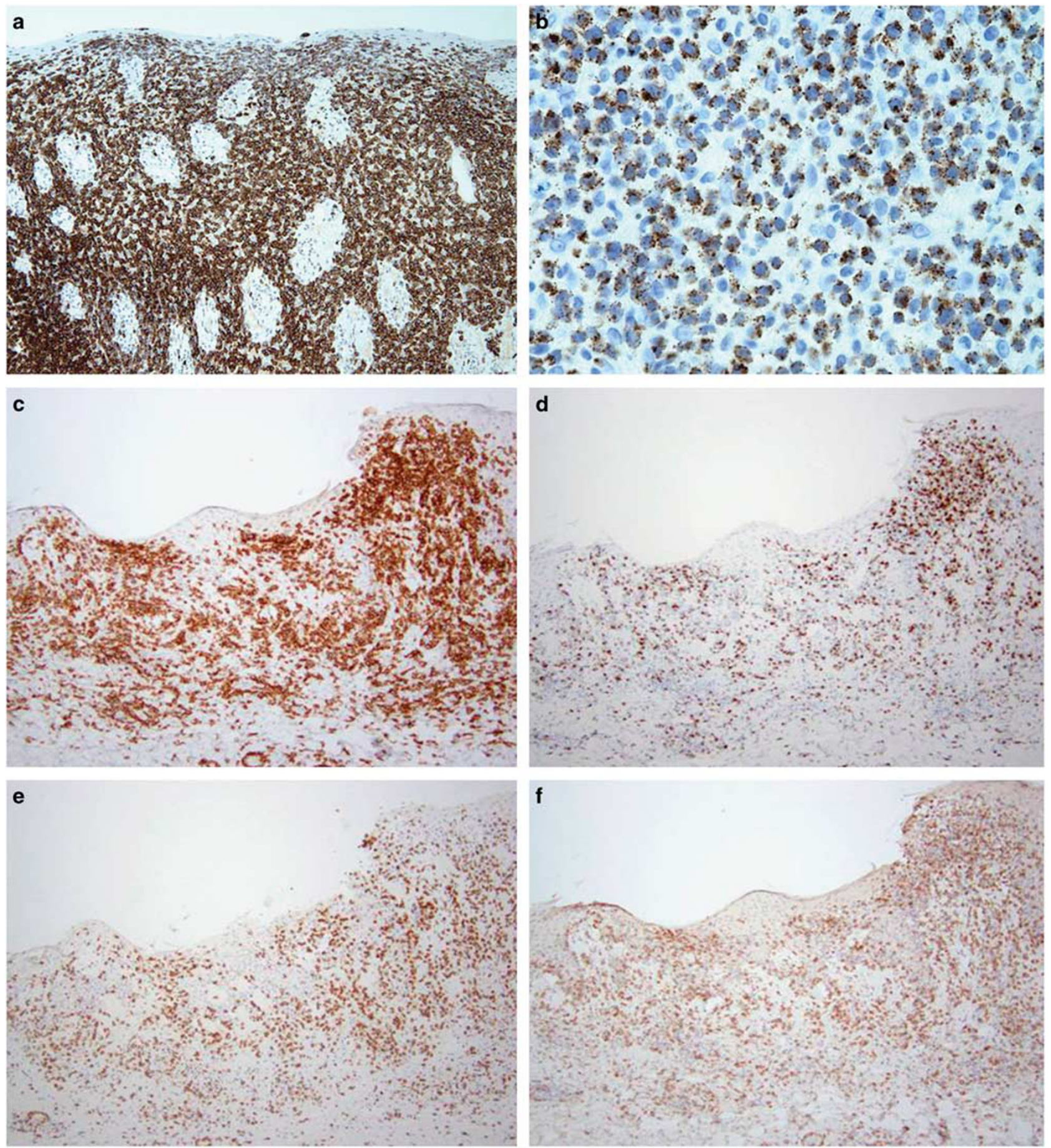

Figure 4 Histopathological features of PCAECyTCL. Immunohistochemistries of case 30 illustrate in 3a with strong CD8 expression (a) and TIA-1 (b). Immunochemistries of case 27 illustrated in figure 1b including CD8 (c), granzyme B (d), and co-expression of $\gamma \delta$ marker (e) and $\beta \mathrm{F} 1(\mathbf{f})$. 
extended beyond the reticular dermis into the subcutaneous tissue (Figure 3e). Focal $(12 / 31)$ or extensive and confluent (8/31) keratinocyte necrosis was noted in $64 \%$ of the biopsies with frank ulceration in seven biopsies. However other biopsies (14/34) showed no signs of epidermal necrosis regardless of lymphoid density. Hence necrosis, hemorrhage, or other histological signs of cytotoxicity may not be observed in some skin biopsies of early evolving lesions. Intraepidermal aggregates of atypical lymphocytes resembling Pautrier's microabscesses were observed in 16/34, more commonly seen in biopsies with high intraepidermal lymphoid density, rather than the scattered microaggregates usually seen in MF. Mild acanthosis was noted in 15 biopsies, but none of the samples had the verrucous hyperplasia so characteristic of pagetoid reticulosis. Although upper dermal extravasation of erythrocytes was common (20/34) often with subtle infiltration of small vessel walls (3/34), fibrinoid vascular necrosis was not noted. However, frank angiocentric or angiodestructive features were not identified in any of the cases. Likewise, modest and focal neurotropism was observed in 5/34 cases. Only one of these biopsies was associated with CD56 (neural cell adhesion molecule) expression and none of them had clinical signs of neuropathy. A minor component of eosinophils in the infiltrate was observed in 13 of the 34 biopsies, including nine with folliculotropism/syringotropism. Few neutrophils $(10 / 34)$ or plasma cells $(2 / 34)$ were noted within the infiltrate in rare biopsies.

The cytological detail of these lymphomas was rather monomorphous and consisted predominantly of medium-sized lymphocytes (24/34). The tumor cells displayed oval or slightly indented nuclei with scant cytoplasm with indistinct cell boundaries (Figure 3f). A predominantly large cell infiltrate was identified in three biopsies.

\section{Immunophenotypic and Molecular Data}

All 34 skin biopsies analyzed with immunohistochemistry were CD3 positive and 34/34 were CD8 positive, whereas seven biopsies had a CD4-/CD8 double-negative phenotype and one of the CD8+ biopsies also showed weak CD4 co-expression (Table 3). We noted CD8 expression to be of variable intensity from case to case (Figure $4 \mathrm{a}$, Case 30). $\beta \mathrm{F} 1$ was positive in $27 / 33$ biopsies. The six $\beta$ F1-negative biopsies were also negative for the $\gamma \delta$ immunomarker. In addition, two biopsies expressed both, the $\gamma \delta$ marker $(\gamma 3.20)$ and the $\alpha \beta$ ( $\beta$ F1) T-cell receptor heterodimers (Figures 4c-f, Case 27). Four of the 30 biopsies tested had partial CD30 positivity, including the two biopsies with predominance of large cells. CD2 was negative in $56 \%(13 / 23)$ of the biopsies. CD56 was positive in $12 \%(4 / 34)$ of the biopsies and CD5 was positive in $33 \%(11 / 33)$ of the biopsies evaluated. CD45RA was expressed in $61 \%(16 / 26)$ of the biopsies and CD7 was positive in $79 \%(23 / 31)$ of the biopsies. Co-expression of CD7 and CD45RA was noted in 12/26 biopsies. Expression of at least one cytotoxic marker including T-cell intracellular antigen-1 (31/32) and granzyme B (22/29) was noted in all biopsies with one exception (Figure 4b, Case 30). Epstein-Barr encoding region in situ hybridization was negative in all 31 biopsies evaluated. T-cell receptor clonality by polymerase chain reaction methods was detected in the skin of $57 \%(4 / 7)$ of the biopsies available for evaluation. T-cell clonality was also detected in the blood of two subjects. Only one case presented with the same clone in the skin and blood by T-cell receptor, which also had a small population by morphology $(6 \%)$.

\section{Clinical or Pathologic Parameters Associated with Survival Outcome}

There was no statistically significant differences in the survival outcome between genders, race, length of disease before diagnosis ( $>6$ months vs $<6$ months), clinical ulcers, or constitutional symptoms at presentation, skin stage T1/ T2 vs T3, number of therapies used, high-serum lactate dehydrogenase, or mucosal membrane involvement (Table 4). A significant worse prognosis was noted in patients presenting with tumors at diagnosis. Extracutaneous involvement did not reach statistical significance, but there were only four patients with it and one of them was alive after allogeneic hematopoietic stem cell transplantation. No statistically significance difference in survival was found associated with large cell (small/medium vs large) or immunophenotypes CD2, CD5, CD7, CD45RA, CD45RA/CD7, CD4-/CD8 -, CD30, granzyme B, and CD56. None of the treatment modalities showed significant survival advantage (Table 2). Allogeneic hematopoietic stem cell transplantation resulted in prolonged partial or complete remissions in five of six patients but did not reach statistical significance.

\section{Discussion}

We have reviewed our experience with a rare and aggressive subtype of cutaneous T-cell lymphoma still considered a provisional entity by the latest World Health Organization classification of cutaneous lymphomas. CD8-positive aggressive epidermotropic T-cell lymphoma has been defined clinically by the abrupt presentation of extensive annular plaques with erosive features and ulceration. ${ }^{6,7}$ This observation reflects the cytotoxic nature of the lymphoma cells, which are embedded primarily in the intraepithelial compartments including epidermis and skin adnexa. The lymphoma cells contain cytotoxic granules, resulting in epidermal necrosis upon activation. The factors that trigger the cytotoxic events are poorly understood, but cases with marked epidermotropism of lymphoma cells without necrotic keratinocytes contrast 
Table 4 Clinical or pathologic parameters associated with survival outcome

\begin{tabular}{|c|c|c|}
\hline Parameter & Cases (dead/total cases) & $\mathrm{P}$-value \\
\hline Gender & Male $10 / 16$ vs female $3 / 8$ & 0.50 \\
\hline Race & Caucasian $8 / 14$ vs others $1 / 6$ & 0.21 \\
\hline Length of disease before diagnosis & $\leq 6 \mathrm{~m} 9 / 12$ vs $>6 \mathrm{~m} \mathrm{4/11}$ & 0.09 \\
\hline Ulcers at presentation & Present 7/15 vs absent 6/9 & 0.69 \\
\hline Constitutional symptoms & Present $5 / 8$ vs absent $5 / 11$ & 0.36 \\
\hline Stage & Т1/T2 1/5 vs Т3 10/16 & 0.11 \\
\hline Total number of therapies & $1-3$ therapies $8 / 11$ vs $4-13$ therapies $4 / 12$ & 0.14 \\
\hline Abnormal LDH & Abnormal $3 / 3$ vs normal $5 / 10$ & 0.16 \\
\hline Mucosal membrane involvement & Present $2 / 5$ vs absent $11 / 19$ & 0.51 \\
\hline Tumors at diagnosis & Present $4 / 4$ vs absent $9 / 20$ & 0.04 \\
\hline Tumors at any time & Present $10 / 15$ vs absent $3 / 9$ & 0.17 \\
\hline Extracutaneous disease & Present $3 / 4$ vs absent $7 / 15$ & 0.31 \\
\hline Large cell (s/m vs large) & $\mathrm{S} / \mathrm{m}$ 9/14 vs large $4 / 10$ & 0.30 \\
\hline CD2 expression & Positive $4 / 7$ vs negative $3 / 10$ & 0.14 \\
\hline CD5 expression & Positive $4 / 10$ vs negative $8 / 13$ & 0.37 \\
\hline CD7 expression & Positive $11 / 19$ vs negative $1 / 4$ & 0.69 \\
\hline CD45RA expression & Positive $7 / 11$ vs negative $3 / 6$ & 0.77 \\
\hline CD45RA/CD7 expression & Positive $6 / 10$ vs negative $7 / 13$ & 0.85 \\
\hline CD4-CD8 - expression & Positive $3 / 5$ vs negative $10 / 19$ & 0.42 \\
\hline CD30 expression & Positive $1 / 3$ vs negative $11 / 20$ & 0.36 \\
\hline Granzyme B expression & Positive $10 / 18$ vs negative $3 / 5$ & 0.95 \\
\hline CD56 expression & Positive $2 / 2$ vs negative $11 / 22$ & 0.23 \\
\hline
\end{tabular}

with other cases with fewer lymphocytes yet with massive keratinocyte necrosis or ulceration. At least in some reported cases, phototherapy and interferon may have coincided with this activation of cytotoxic events. ${ }^{8}$ Angioinvasion and angiodestructive features have been occasionally reported in CD8positive aggressive epidermotropic T-cell lymphoma. ${ }^{7,9}$ Although subtle involvement of dermal vasculature by the lymphoma cells was observed in a few cases, ulceration seems to be the direct result of epithelial necrosis from cytotoxic lymphocytes rather than a consequence of vaso-occlusion or angiodestruction as seen in natural killer T-cell lymphomas associated with Epstein-Barr virus infection. This pathomechanism of massive epidermal necrosis triggered by a clonal expansion of cytotoxic T-cells is also shared by pityriasis lichenoides acuta or Mucha-Habermann disease. Hence, it comes as no surprise that the febrile ulceronecrotic variant of Mucha-Habermann can pose a diagnostic dilemma, as observed in two of our cases. By definition, extensive epidermotropism is a diagnostic requirement, but in some cases the epidermal lymphocytes remained localized in the lower epidermal layers. Furthermore, the burden of tumor cells may be dermal in thick plaque or tumor lesions with pagetoid extension into the surmounting epidermis.

As reported by Robson et al., despite extensive adnexotropism, clinical evidence of folliculotropism including follicular-based papules (keratosis pilaris, lichen spinulosus, milia, or cystic lesions) or alopecia mucinosa were not observed in our series. ${ }^{7}$ In addition, destruction of adnexal structures with resulting granulomatous reaction or mucinous degeneration as seen in folliculotropic mycosis fungoides do not seem to occur in primary cutaneous aggressive epidermotropic cytotoxic T-cell lymphoma.

Unlike other cytotoxic lymphomas such as $\gamma \delta$ T-cell lymphomas that are often associated with immune dysregulation or natural killer T-cell lymphomas associated with Epstein-Barr virus infection, we have not identified any significant comorbidities or predisposing factors associated with primary cutaneous aggressive epidermotropic cytotoxic T-cell lymphoma. ${ }^{10}$ However, preceding skin signs of poorly defined papulo-squamous or eczematous dermatitis were observed in a several patients. The clinical impression of these preceding lesions varied from psoriasis to eczema or mycosis fungoides. We had the opportunity to review skin biopsies of preceding lesions of two cases, which showed psoriasiform acanthosis with subtle scattered intraepidermal atypical lymphocytes in one case and folliculotropism and syringotropism with eosinophils in another case resembling adnexotropic mycosis fungoides. Previous reports of similar epidermotropic cytotoxic lymphomas initially misinterpreted as 'ulcerative psoriasis' also emphasized the fact that a lymphoid-rich skin biopsy should be a warning sign not compatible with psoriasis vulgaris. ${ }^{11}$ In addition, we noted scattered necrotic keratinocytes in these early lesion biopsies, a finding rarely seen in psoriasis or mycosis fungoides.

As initially reported by the European group, the World Health Organization defines CD8-positive aggressive epidermotropic T-cell lymphoma by the expression of CD8. However, we have observed cases with CD4/CD8 double negativity that otherwise fulfill clinical and pathological characteristics of this entity. Likewise, CD45RA, a marker commonly 
expressed in CD8-positive aggressive epidermotropic T-cell lymphoma was negative or partially positive in many cases and hence not a reliable diagnostic marker. The variable CD45RA expression is not surprising as the marker is reversible with stronger expression in resting and naive lymphocytes when antigenic stimulation is low. ${ }^{12}$ In addition, we observed variable expression of the T-cell receptor heterodimer markers. A small subset of cutaneous lymphomas with similar clinicopathological presentation expressing only $\gamma \delta$ T-cell receptor heterodimer with $\alpha \beta$ negativity were reviewed and excluded for this study. However, a few other cases with $\alpha \beta / \gamma \delta$ double-positive or double-negative expression were included. This unexpected variable expression of T-cell receptor heterodimers ranging from $\alpha \beta / \gamma \delta$ T-cell receptor double positivity to double negativity has also been reported in natural killer T-cell lymphomas. ${ }^{13}$ This observation may not be entirely surprising as the $\gamma \delta$ immunomarkers for paraffinembedded tissue were not widely available when Berti et al. published their initial case series. ${ }^{14}$

A recent publication by Merrill et al. highlighted aggressive primary cutaneous gamma delta T-cell lymphoma presenting with pagetoid epidermotropism. ${ }^{15}$ We considered this diagnostic possibility for the two cases expressing the gamma T-cell receptor in addition to the beta T-cell receptor marker (cases 22 and 27). However, we felt the clinical and pathological findings of these cases was most consistent with the rest of the primary cutaneous aggressive epidermotropic cytotoxic T-cell lymphoma cohort and as previously mentioned variable expression of alpha/beta and gamma/ delta markers is occasionally observed in other cytototxic lymphomas. Based on our observation, we recommend the term primary cutaneous aggressive epidermotropic cytotoxic T-cell lymphoma, to include not only the previously described CD8+/Tcell receptor- $\beta+$ cases but the additional CD4/CD8 double-negative cases and/or cases with variable $\alpha \beta$ / $\gamma \delta$ heterodimer expression, as long as at least one T-cell marker and one marker of cytotoxicity (i.e. T-cell intracellular antigen-1, perforin, or granzyme B) is expressed.

Our cohort confirms the poor prognosis of primary cutaneous aggressive epidermotropic cytotoxic T-cell lymphoma with rapid disease progression, poor response to chemotherapy and death often associated with complications of extensive epidermal necrosis and only rarely nodal or other extracutaneous involvement. In contrast to other cytotoxic lymphomas, hemophagocytic lymphohistiocytosis was not observed in any of our cases. We found one case report of primary cutaneous aggressive epidermotropic cytotoxic T-cell lymphoma complicated with hemophagocytic lymphohistiocytosis in the literature, but the diagnosis of this human immunodeficiency virus-positive patient with Epstein-Barr virus expression in the intraepidermal lymphoid cells is questionable. ${ }^{16}$
Besides the rare and equally aggressive epidermotropic variants of cutaneous $\gamma \delta$ T-cell lymphomas, the differential diagnosis of primary cutaneous aggressive epidermotropic cytotoxic T-cell lymphoma includes CD8-positive mycosis fungoides, localized pagetoid reticulosis (Woringer-Kolopp disease) and type D lymphomatoid papulosis. These three indolent conditions can occasionally present with a similar complete or partial CD45RA+/ CD7+/CD8+ phenotype profile. ${ }^{7,12}$ The histopathology of mycosis fungoides tends to be less pagetoid with more pleomorphic and convoluted nuclei and most importantly without necrotic keratinocytes. Intraepidermal aggregates of lymphocytes resembling Pautrier microabscesses were a common finding and hence may not help distinguish from mycosis fungoides. Localized pagetoid reticulosis (Woringer-Kolopp disease) and type D lymphomatoid papulosis can have similar biopsy findings including epidermal necrosis, but usually the clinical presentation does not pose a diagnostic dilemma. Nevertheless, one of our patients initially presented with a single lesion and the diagnosis of Woringer-Kolopp disease was considered, but extensive patches appeared shortly after.

Based on our experience, we feel that primary cutaneous aggressive epidermotropic cytotoxic T-cell lymphoma should be defined clinically by the rapid onset of erosive or ulcerated plaques with or without preceding skin lesions and should not be limited to immunophenotypic expression of CD8 or the $\alpha \beta$ T-cell receptor heterodimer, but rather the identification of an atypical pagetoid T-cell infiltrate with cytotoxic granules and absence of CD4 expression. Molecular studies should demonstrate dominant T-cell receptor sequences. We also bring attention to the yet poorly defined preceding skin lesions reported in several of our patients and often misdiagnosed as psoriasis, eczema, or mycosis fungoides. It remains an important goal to learn to recognize and diagnose these early stages. Tracking the evolution of T-cell clones with high throughput sequencing may allow us to better understand these precursor lesions. Although there is no evidence that early intervention will prevent the development of extensive ulceration with high morbidity, identification of these early stages may alert us to patients that should be closely followed avoiding immunosuppressive therapies, which could accelerate the course, as seen in some of our patients. Finally, our series highlights the failure of most standard cutaneous T-cell lymphoma therapies in treating this condition. In our experience, allogeneic hematopoietic stem cell transplantation appears to be the only therapeutic option associated with durable partial or complete remission raising the question of whether this treatment should be considered early on and prior to the development of extensive epidermal necrosis and ulceration which is often associated with a poor outcome. However, the scarcity of reports of primary cutaneous aggressive 
epidermotropic cytotoxic T-cell lymphoma still precludes us from establishing therapeutic guidelines.

\section{Acknowledgments}

This research was funded in part through the NIH/ NCI Cancer Center Support (Grant P30 CA008748), TCF Foundation, Plymouth MN, Therakos.

\section{Disclosure/conflict of interest}

The authors declare no conflict of interest.

\section{References}

1 Swerdlow SH, Campo E, Pileri SA, et al. The 2016 revision of the World Health Organization classification of lymphoid neoplasms. Blood 2016;127:2375-2390.

2 Agnarsson BA, Vonderheid EC, Kadin ME. Cutaneous T cell lymphoma with suppressor/cytotoxic (CD8) phenotype: identification of rapidly progressive and chronic subtypes. J Am Acad Dermatol 1990;22: 569-577.

3 Berti E, Tomasini D, Vermeer $\mathrm{MH}$, et al. Primary cutaneous CD8-positive epidermotropic cytotoxic T cell lymphomas. A distinct clinicopathological entity with an aggressive clinical behavior. Am J Pathol 1999;155:483-492.

4 Gormley RH, Hess SD, Anand D, et al. Primary cutaneous aggressive epidermotropic CD8+ T-cell lymphoma. J Am Acad Dermatol 2010;62:300-307.

5 Kim YH, Willemze R, Pimpinelli N, et al. TNM classification system for primary cutaneous lymphomas other than mycosis fungoides and Sezary syndrome: a proposal of the International Society for Cutaneous Lymphomas (ISCL) and the Cutaneous Lymphoma Task Force of the European Organization of Research and Treatment of Cancer (EORTC). Blood 2007;110:479-484.

6 Nofal A, Abdel-Mawla MY, Assaf M, et al. Primary cutaneous aggressive epidermotropic CD8+ T-cell lymphoma: proposed diagnostic criteria and therapeutic evaluation. J Am Acad Dermatol 2012;67:748-759.
7 Robson A, Assaf C, Bagot M, et al. Aggressive epidermotropic cutaneous CD8+ lymphoma: a cutaneous lymphoma with distinct clinical and pathological features. Report of an EORTC Cutaneous Lymphoma Task Force Workshop. Histopathology 2015;67:425-441.

8 Paulli M, Berti E. Cutaneous T-cell lymphomas (including rare subtypes). Current concepts. II. Haematologica 2004;89:1372-1388.

9 Wang Y, Li T, Tu P, et al. Primary cutaneous aggressive epidermotropic CD8+ cytotoxic T-cell lymphoma clinically simulating pyoderma gangrenosum. Clin Exp Dermatol 2009;34:e261-e262.

10 Guitart J, Weisenburger DD, Subtil A, et al. Cutaneous gammadelta T-cell lymphomas: a spectrum of presentations with overlap with other cytotoxic lymphomas. Am J Surg Pathol 2012;36:1656-1665.

11 Weenig RH, Comfere NI, Gibson LE, et al. Fatal cytotoxic cutaneous lymphoma presenting as ulcerative psoriasis. Arch Dermatol 2009;145:801-808.

12 Fierro MT, Novelli M, Savoia P, et al. CD45RA+ immunophenotype in mycosis fungoides: clinical, histological and immunophenotypical features in 22 patients. J Cutan Pathol 2001;28:356-362.

13 Pongpruttipan T, Sukpanichnant S, Assanasen T, et al. Extranodal NK/T-cell lymphoma, nasal type, includes cases of natural killer cell and alphabeta, gammadelta, and alphabeta/gammadelta T-cell origin: a comprehensive clinicopathologic and phenotypic study. Am J Surg Pathol 2012;36:481-499.

14 Roullet M, Gheith SM, Mauger J, et al. Percentage of \{gamma\}\{delta\} $\mathrm{T}$ cells in panniculitis by paraffin immunohistochemical analysis. Am J Clin Pathol 2009;131:820-826.

15 Merrill ED, Agbay R, Miranda RN, et al. Primary cutaneous T-cell lymphomas showing gamma-delta (gammadelta) phenotype and predominantly epidermotropic pattern are clinicopathologically distinct from classic primary cutaneous gammadelta T-cell lymphomas. Am J Surg Pathol 2016.

16 Karkouche R, Ingen-Housz-Oro S, Le Gouvello S, et al. Primary cutaneous aggressive epidermotropic CD8+ T-cell lymphoma with KIR3DL2 and NKp46 expression in a human immunodeficiency virus carrier. J Cutan Pathol 2015;42:199-205. 